\title{
Endosonographic Features of Gastric Schwannoma: A Single Center Experience
}

\author{
Jong Min Yoon ${ }^{1}$, Gwang Ha Kim¹, Do Youn Park², Na Ri Shin², Sangjeong Ahn², Chul Hong Park', Jin Sung Lee', Key Jo Lee', \\ Bong Eun Lee ${ }^{1}$ and Geun Am Song ${ }^{1}$ \\ Departments of ${ }^{1}$ Internal Medicine and ${ }^{2}$ Pathology, Pusan National University School of Medicine, Busan, Korea
}

Background/Aims: Gastric schwannomas are rare benign mesenchymal tumors that are difficult to differentiate from other mesenchymal tumors with malignant potential, such as gastrointestinal stromal tumors. This study aimed to evaluate the characteristic findings of gastric schwannomas via endoscopic ultrasonography (EUS).

Methods: We retrospectively reviewed the EUS findings of 27 gastric schwannoma cases that underwent surgical excision at Pusan National University Hospital during 2007 to 2014.

Results: Gastric schwannomas were mainly located in the middle third of the stomach with a mean tumor size of $32 \mathrm{~mm}$. All lesions exhibited hypoechoic echogenicity, and 24 lesions (88.9\%) exhibited heterogeneous echogenicity. Seventeen lesions (63.0\%) exhibited decreased echogenicity compared to the normal proper muscle layer. Distinct borders were observed in 24 lesions (88.9\%), lobulated margins were observed in six lesions (22.2\%), and marginal haloes were observed in 24 lesions (88.9\%). Hyperechogenic spots were observed in 21 lesions (77.8\%), calcifications were observed in one lesion (3.7\%), and cystic changes were observed in two lesions (7.4\%). Conclusions: During EUS, gastric schwannomas appear as heterogeneously hypoechoic lesions with decreased echogenicity compared to the normal proper muscle layer. These features may be helpful for differentiating gastric schwannomas from other mesenchymal tumors. Clin Endosc 2016;49:548-554

Key Words: Endosonography; Mesenchymal tumor; Schwannoma; Stomach

\section{INTRODUCTION}

Gastric mesenchymal tumors are usually found incidentally during endoscopy for unrelated conditions, and appear as firm, protruding subepithelial masses. The entities that are responsible for mesenchymal tumors include leiomyomas, gastrointestinal stromal tumors (GISTs), and schwannomas. Among these entities, GISTs are the most common gastric

Received: September 10, 2015 Revised: December 1, 2015

Accepted: December 5, 2015

\section{Correspondence: Gwang Ha Kim}

Department of Internal Medicine, Pusan National University Hospital, Pusan National University School of Medicine, 179 Gudeok-ro, Seo-gu, Busan 49241, Korea

Tel: +81-51-240-7869, Fax: +81-51-244-8180, E-mail: doc0224@pusan.ac.kr

cc This is an Open Access article distributed under the terms of the Creative Commons Attribution Non-Commercial License (http://creativecommons.org/ licenses/by-nc/3.0) which permits unrestricted non-commercial use, distribution, and reproduction in any medium, provided the original work is properly cited. mesenchymal tumors that have a malignant potential. ${ }^{1,2}$

Schwannomas are tumors of spindle cells that originate from any nerve having a Schwann cell sheath, which are also known as neurinomas or neurilemmomas. Schwannomas in the gastrointestinal tract are rare (approximately $3 \%$ of all gastrointestinal mesenchymal tumors), and typically occur in the stomach. ${ }^{3,4}$ Gastric schwannomas arise from the sheath of Auerbach's plexus or, less frequently, from Meissner's plexus. ${ }^{5}$ Schwannomas are generally benign, usually asymptomatic, slow-growing mesenchymal tumors with a very low malignant potential, and they have an excellent prognosis after surgical resection. ${ }^{3}$ However, these tumors are often misdiagnosed as GISTs. It is therefore important to accurately differentiate schwannomas from GISTs.

Endoscopic ultrasonography (EUS) is a valuable imaging modality for diagnosing and evaluating gastric mesenchymal tumors, because they appear as hypoechoic masses contiguous with the proper muscle layer of the normal gut wall on EUS. ${ }^{6}$ 
Although a definitive diagnosis of a gastric mesenchymal tumor is made via microscopic examination and immunohistochemical staining, ${ }^{3}$ the evaluation of EUS findings regarding tumor size or pattern of echogenicity can be helpful in the differential diagnosis of mesenchymal tumors. However, only a few studies have examined the characteristic EUS findings of gastric schwannomas. ${ }^{7-9}$ Thus, it is important to develop a better understanding of the EUS features of gastric schwannomas; differentiating them from other mesenchymal tumors (especially GISTs) can prevent inappropriate surgery and facilitate the use of minimal invasive treatment. Therefore, the present study aimed to evaluate the characteristic EUS findings of gastric schwannoma.

\section{MATERIALS AND METHODS}

A database of all patients who underwent EUS at Pusan National University Hospital (Busan, Korea) between January 2007 and December 2014 was retrospectively analyzed. We identified 27 patients who were histopathologically confirmed to have gastric schwannoma after surgical resection. This study's design was reviewed and approved by the Institutional Review Board at Pusan National University Hospital (E-2015009), and informed consent was obtained from all patients before they underwent the original treatment.

\section{Endoscopic ultrasonography}

EUS was performed with a radial scanning ultrasound endoscope (GF-UM2000; Olympus, Tokyo, Japan) at 7.5 and 12 $\mathrm{MHz}$. All examinations were performed under intravenous conscious sedation (using midazolam with or without meperidine), and the tumors were scanned after filling the stomach with 400 to $800 \mathrm{~mL}$ of deaerated water. At least 10 still images were obtained for each lesion during EUS, and digital copies of these images were saved in our database.

In this study, the EUS images were reviewed by a single experienced endosonographer (GHK). The following EUS features were analyzed: location; gross shape using the Yamada classification; ${ }^{10}$ the presence of mucosal ulceration during endoscopy and/or EUS; maximal diameter; growth pattern (intraluminal, mural, or extraluminal); sonographic layer of origin; echogenicity compared to the surrounding normal proper muscle layer (increased or decreased); homogeneity (homogenous or heterogeneous); distinctness of the borders (distinct or indistinct); the presence of marginal haloes and lobulated margins; and the presence of cystic change and calcification.

\section{Histopathological evaluation}

Hematoxylin and eosin slides were reviewed for all cases, and the histological features (mitotic count per 50 high power fields and the presence of lymphoid cuff) were recorded. For the immunohistochemical assessments, the following antibodies were used after pretreatment for epitope retrieval: anti-ckit (P145; Novocastra, Newcastle, UK), anti-CD34 (QBEnd-10; DAKO, Carpinteria, CA, USA), anti- $\alpha$-smooth muscle actin (SMA) (1A4; DAKO), anti-desmin (D33; DAKO), anti-S-100 protein (S100P; Novocastra), and anti-Ki-67 (MIB-1, DAKO). Schwannoma was defined as an S-100-positive and c-kit-negative tumor, leiomyoma was defined as a desmin-positive or SMA-positive and c-kit-negative tumor, and GIST was defined as a c-kit-positive tumor. ${ }^{11}$ The Ki-67 labeling index was calculated as the number of positive nuclear reactions per 100 cells.

\section{RESULTS}

\section{Clinical characteristics of patients with gastric schwannoma}

The 27 patients comprise 11 men and 16 women, with a mean age of 53 years (range, 26 to 68). Twenty-three patients (85\%) had no symptoms, and their gastric schwannomas were incidentally detected during endoscopic screening. The four remaining patients (15\%) were symptomatic, and presented with dyspepsia or epigastric pain (Table 1). All patients were treated via either laparoscopic wedge resection $(n=24)$ or subtotal gastrectomy $(n=3)$. Three patients underwent subtotal gastrectomy due to large tumor size $(n=1)$ or concurrent gastric cancer $(n=2)$. None of the patients had a history of neurofibromatosis syndrome.

\section{EUS findings of gastric schwannoma}

Seventeen lesions (63\%) were located in the middle third, eight lesions (30\%) were located in the upper third, and two lesions (7\%) were located in the lower third of the stomach. Three lesions (11\%) exhibited surface ulceration, and almost all lesions $(25 / 27,93 \%)$ were located in the fourth (proper muscle) layer (Table 2). The lesions had a mean size of 32 $\mathrm{mm}$ (range, 13 to 88). Fifteen lesions (56\%) exhibited a mural growth pattern, and 12 lesions (44\%) exhibited an extraluminal growth pattern. All lesions exhibited hypoechoic echogenicity, 24 lesions (89\%) were heterogeneous, and distinct borders were observed in 24 lesions (89\%). Compared to the echogenicity of the surrounding normal proper muscle layer, 17 lesions (63\%) exhibited decreased echogenicity and 10 lesions (37\%) exhibited increased echogenicity (Fig. 1). Lobulated margins were observed in six lesions (22\%), and marginal haloes were observed in 24 lesions (89\%). Hyperechogenic 
$C_{\text {cunwche Enooscopy }}$

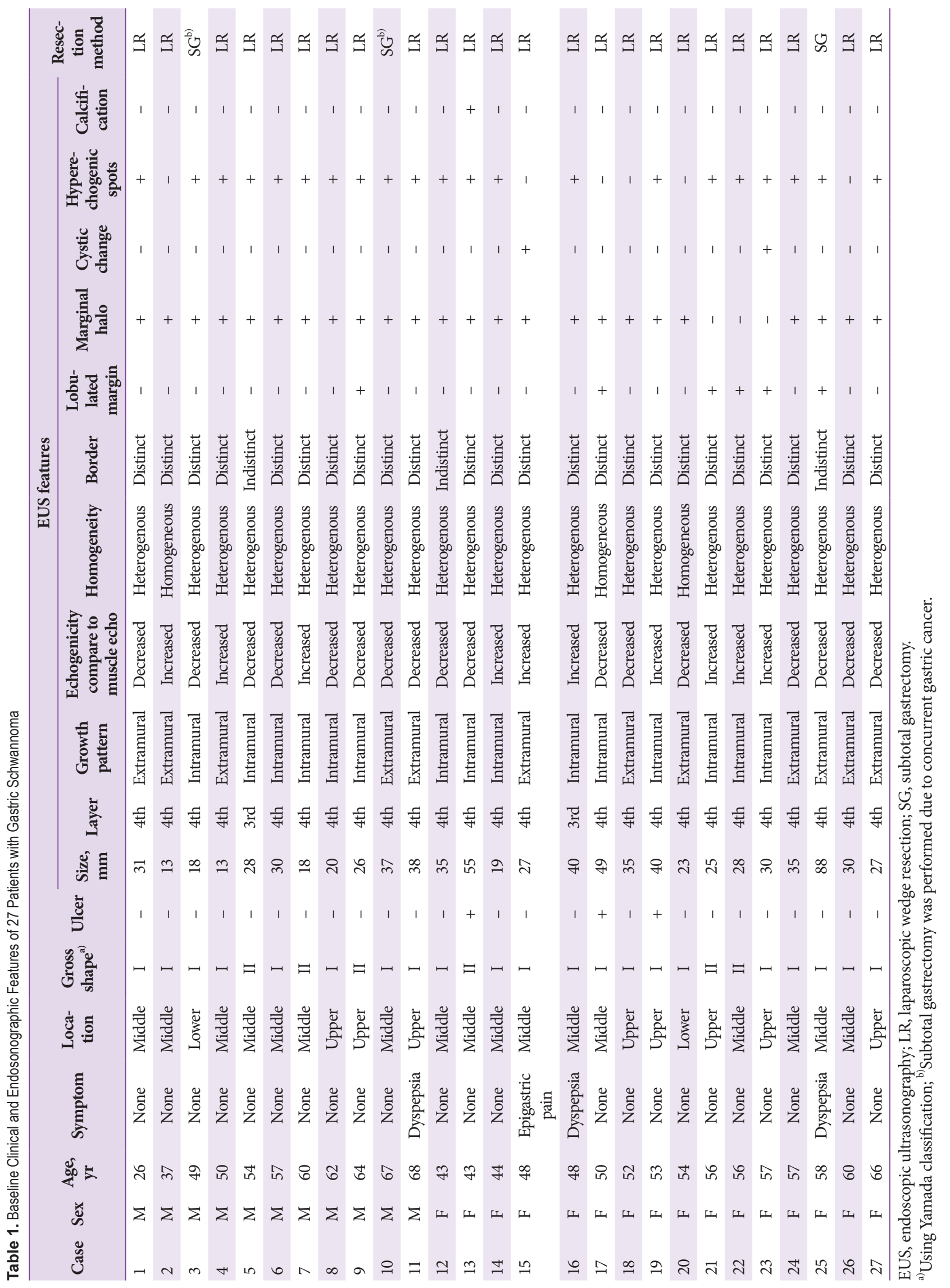


Table 2. Endosonographic Features of 27 Patients with Gastric Schwannoma

\begin{tabular}{|c|c|}
\hline Endosonographic feature & No. $(\%)$ \\
\hline \multicolumn{2}{|l|}{ Location } \\
\hline Upper third & $8(29.6)$ \\
\hline Middle third & $17(63.0)$ \\
\hline Lower third & $2(7.4)$ \\
\hline \multicolumn{2}{|l|}{ Ulcer } \\
\hline Absent & $24(88.9)$ \\
\hline Present & $3(11.1)$ \\
\hline Size, mm, mean (range) & $32(13-88)$ \\
\hline \multicolumn{2}{|l|}{ Layer of origin } \\
\hline Third layer & $2(7.4)$ \\
\hline Fourth layer & $25(92.6)$ \\
\hline \multicolumn{2}{|l|}{ Growth pattern } \\
\hline Mural & $15(55.6)$ \\
\hline Extraluminal & $12(44.4)$ \\
\hline \multicolumn{2}{|c|}{ Echogenicity vs. the surrounding muscle echo } \\
\hline Decreased & $17(63.0)$ \\
\hline Increased & $10(37.0)$ \\
\hline \multicolumn{2}{|l|}{ Homogeneity } \\
\hline Homogenous & $3(11.1)$ \\
\hline Heterogeneous & $24(88.9)$ \\
\hline \multicolumn{2}{|l|}{ Border } \\
\hline Distinct & $24(88.9)$ \\
\hline Indistinct & $3(11.1)$ \\
\hline \multicolumn{2}{|l|}{ Lobulated margin } \\
\hline Absent & $21(77.8)$ \\
\hline Present & $6(22.2)$ \\
\hline \multicolumn{2}{|l|}{ Marginal halo } \\
\hline Absent & $3(11.1)$ \\
\hline Present & $24(88.9)$ \\
\hline \multicolumn{2}{|l|}{ Cystic change } \\
\hline Absent & $25(92.6)$ \\
\hline Present & $2(7.4)$ \\
\hline \multicolumn{2}{|l|}{ Hyperechogenic spot } \\
\hline Absent & $6(22.2)$ \\
\hline Present & $21(77.8)$ \\
\hline \multicolumn{2}{|l|}{ Calcification } \\
\hline Absent & $26(96.3)$ \\
\hline Present & $1(3.7)$ \\
\hline
\end{tabular}

spots were observed in 21 lesions (78\%), calcification was observed in one lesion (4\%), and cystic changes were observed in two lesions (7\%).

\section{Histopathological findings}

Evaluation of the resected specimens revealed that the tumors were composed of haphazardly arranged spindle cells. Cuff-like lymphoid infiltration at the tumor periphery was observed in 26 tumors (Table 3 ). The mitotic rate was $<5$ mitoses per 50 high power fields in all tumors. All tumors were positive for S-100 protein (Fig. 1), and were negative for c-kit and desmin. Twenty-five tumors were negative for CD34 and SMA, one tumor was positive for CD34, and another tumor was positive for SMA. The Ki-67 labeling index did not exceed $5 \%$ in all tumors.

\section{DISCUSSION}

In clinical practice, it is difficult to preoperatively differentiate between schwannomas and GISTs, even if EUS-guided performed. ${ }^{12-14}$ Thus, identifying characteristic findings for schwannomas via non-invasive imaging techniques would be helpful for treating patients with gastric mesenchymal tumors. Here, we investigated the characteristic EUS features of gastric schwannomas using EUS, which is useful for diagnosing subepithelial lesions. Our findings indicate that gastric schwannomas were heterogeneously hypoechoic lesions with distinct borders, marginal haloes, and decreased echogenicity compared to that of the surrounding normal proper muscle layer.

Gastric schwannomas occur most frequently during the fourth and fifth decades of life, and predominantly in women. ${ }^{15}$ Because most patients with gastric schwannoma are asymptomatic, these lesions are usually diagnosed incidentally during upper endoscopy for unrelated symptoms. However, large tumors or tumors with mucosal ulcerations can occasionally cause bleeding. ${ }^{11}$ Similarly, we observed a female:male ratio of 1.5:1 and a mean patient age of 53 years. Furthermore, only four of our patients (15\%) were symptomatic, and their symptoms were epigastric pain or dyspepsia.

Computed tomography (CT), magnetic resonance imaging (MRI), and positron emission tomography (PET) provide limited information for differentiating schwannomas from other mesenchymal tumors in the stomach. For example, previous CT studies have demonstrated that gastric schwannomas were well-circumscribed masses showing homogeneous or heterogeneous contrast enhancement. ${ }^{16,17}$ In addition, MRI studies have revealed that gastric schwannomas were well demarcated and strongly enhanced tumors, with low-to-medium signal intensity on T1-weighted images and high signal intensity on T2-weighted images. ${ }^{18}$ However, these radiological findings for gastric schwannomas are not specific, and are very similar to those of GISTs. Furthermore, several recent reports have 

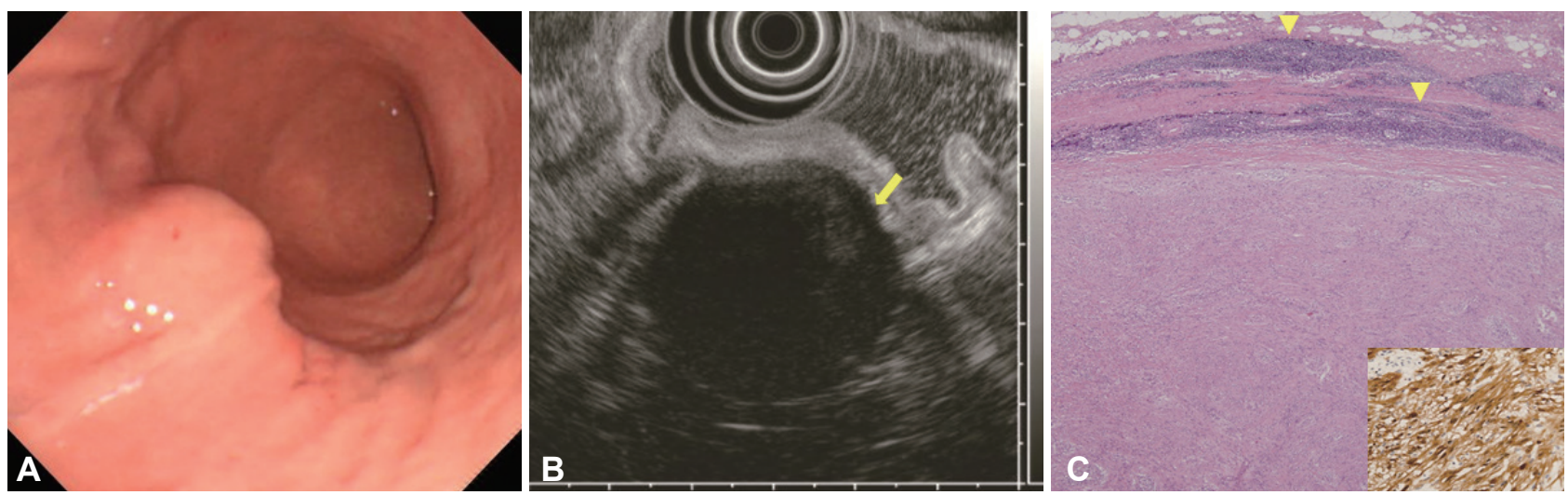

Fig. 1. A case of gastric schwannoma (case 24). (A) Endoscopy reveals a subepithelial mass in the middle third of the stomach. (B) Endoscopic ultrasonography reveals that the mass is heterogeneous with an echogenicity lower than that of the surrounding normal proper muscle layer. The lesion size is $35 \times 30 \mathrm{~mm}$, and marginal haloes (arrow) are visible. (C) Microscopic examination reveals a relatively circumscribed mass that is composed of variably organized spindle cells surrounded by patches of lymphoid infiltration (arrowheads; H\&E stain, $\times 40$ ). The tumor cells are strongly positive for S-100 protein (boxed area, anti-S-100 antibody stain, $\times 400$ ).

showed gastric schwannomas with increased fluorodeoxyglucose uptake during PET, which is similar to the findings for gastric GISTs. ${ }^{18,19}$

Although EUS is a useful imaging technique for diagnosing and evaluating gastric mesenchymal tumors, only a few reports have been published regarding the EUS characteristics of gastric schwannomas because of the rarity of these tumors. For example, EUS studies have described gastric schwannomas as round homogenous masses with marginal haloes and without internal echogenic foci. ${ }^{7,20}$ In addition, the echogenicity of gastric schwannomas was lower than that of the surrounding normal muscle layers. In other studies, EUS evaluation of gastric schwannomas revealed heterogeneous and hypoechoic masses with some hyperechogenic foci. ${ }^{8,9}$ In the present study, most of the gastric schwannomas exhibited heterogeneous and hypoechoic echogenicity with internal hyperechogenic foci and marginal haloes.

In the present study, we paid careful attention to the tumor echogenicity because almost all gastric mesenchymal tumors appear as hypoechoic lesions during EUS. We therefore compared the echogenicity of the tumor to that of the surrounding normal proper muscle layer. In our previous study, although $>50 \%$ of the GISTs exhibited increased echogenicity, compared to the surrounding normal proper muscle layer, the echogenicity of leiomyomas was similar to that of the surrounding normal proper muscle layer. ${ }^{6}$ In contrast, our present study revealed that two-thirds of the schwannoma exhibited lower echogenicity, compared to the surrounding normal proper muscle layer. This finding is consistent with the findings of our previous study regarding digital image analysis after standardized EUS images for gastric mesenchymal tumors, which revealed that the intensity values for schwannomas were lower than those for GISTs. ${ }^{1}$

Interestingly, it has also been reported that EUS findings reflect the pathologic features of gastric schwannoma. ${ }^{7,20}$ For example, the trend towards a lower echogenicity for schwannomas in the present study may be related to a reduction in acoustic impedance because of the dense composition of spindle cells. ${ }^{21}$ In addition, approximately $90 \%$ of the gastric schwannomas in the present study had marginal haloes, which may correspond to the peripheral lymphoid cuff in our histological analysis. ${ }^{20}$ Nevertheless, despite marginal haloes being extremely common among schwannomas, this feature is not specific to schwannomas, and is frequently observed in GISTs. $^{6}$

Previous EUS studies have also suggested that exogastric growth, cystic changes within the tumor, hyperechogenic foci in the tumor, and lobulation of the tumor margins favor the diagnosis of malignant mesenchymal tumors, such as GISTs. ${ }^{22-24}$ Our results indicated that these features might also be useful in the identification of gastric schwannomas, as extraluminal growth and hyperechogenic foci within the tumor were common for these lesions, although cystic changes and lobulated margins were uncommon.

A definitive diagnosis of a gastric mesenchymal tumor is typically confirmed via microscopic histopathological examination and immunohistochemical staining. Tissue acquisition can be obtained via EUS-guided tissue sampling, endoscopic resection, or surgical resection. These tumors are typically negative for c-kit, desmin, and SMA; are typically positive for S-100; and stain variably for CD $34 .{ }^{25}$ In the present study, all cases were negative for c-kit and positive for S-100, and most cases (25 of 27) were negative for desmin, SMA, and $\mathrm{CD} 34$. However, the diagnostic accuracy of EUS-guided tissue sampling was only $\sim 50 \%$ to $60 \%$, due to technical failure and inadequate sampling. ${ }^{26}$ Therefore, we believe that our results regarding the EUS features of gastric schwannoma may be useful for managing patients with gastric mesenchymal tu- 
Table 3. Histopathological Findings from 27 Patients with Gastric Schwannoma

\begin{tabular}{|c|c|c|c|c|c|c|c|c|}
\hline \multirow{2}{*}{ Case } & \multirow{2}{*}{$\begin{array}{c}\text { Mitoses/50 } \\
\text { HPFs }\end{array}$} & \multirow{2}{*}{$\begin{array}{l}\text { Lymphoid } \\
\text { cuff }\end{array}$} & \multicolumn{6}{|c|}{ Immunohistochemical results } \\
\hline & & & c-kit & CD34 & SMA & Desmin & S-100 & Ki-67 labelling index, \% \\
\hline 1 & 0 & + & - & - & - & - & + & $<1$ \\
\hline 2 & 0 & + & - & - & - & - & + & $<1$ \\
\hline 3 & 0 & + & - & - & - & - & + & $2-5$ \\
\hline 4 & 0 & + & - & - & - & - & + & $2-5$ \\
\hline 5 & 1 & + & - & - & - & - & + & $2-5$ \\
\hline 6 & 0 & + & - & - & - & - & + & $<1$ \\
\hline 7 & 2 & + & - & - & - & - & + & $2-5$ \\
\hline 8 & 1 & + & - & - & - & - & + & $2-5$ \\
\hline 9 & 0 & + & - & - & - & - & + & $<1$ \\
\hline 10 & 1 & + & - & - & + & - & + & $2-5$ \\
\hline 11 & 0 & + & - & - & - & - & + & $<1$ \\
\hline 12 & 0 & + & - & - & - & - & + & $<1$ \\
\hline 13 & 0 & + & - & - & - & - & + & $<1$ \\
\hline 14 & 0 & + & - & - & - & - & + & $<1$ \\
\hline 15 & 0 & + & - & - & - & - & + & $<1$ \\
\hline 16 & 0 & + & - & - & - & - & + & $2-5$ \\
\hline 17 & 1 & + & - & - & - & - & + & $2-5$ \\
\hline 18 & 1 & + & - & - & - & - & + & $2-5$ \\
\hline 19 & 1 & + & - & - & - & - & + & $2-5$ \\
\hline 20 & 0 & + & - & - & - & - & + & $2-5$ \\
\hline 21 & 1 & + & - & - & - & - & + & $2-5$ \\
\hline 22 & 1 & + & - & - & - & - & + & $2-5$ \\
\hline 23 & 1 & - & - & + & - & - & + & $<1$ \\
\hline 24 & 1 & + & - & - & - & - & + & $<1$ \\
\hline 25 & 1 & + & - & - & - & - & + & $2-5$ \\
\hline 26 & 0 & + & - & - & - & - & + & $2-5$ \\
\hline 27 & 0 & + & - & - & - & - & + & $<1$ \\
\hline
\end{tabular}

HPF, high power field; SMA, smooth muscle actin.

mors.

This study had several limitations. Firstly, we used a retrospective design to identify the EUS features of gastric schwannomas, and there might have been bias during the retrospective review of the EUS images. However, at least 10 images were obtained during EUS examination, and this may partially compensate for any bias that was related to the retrospective design. Secondly, although EUS examinations were performed, the patients were only selected for surgery based on the clinical opinion and decision of their physician. Finally, the number of gastric schwannomas included in the present study is somewhat small, because schwannomas are relatively rare lesions in the stomach compared to GISTs. Therefore, prospective multi-center studies are needed to validate our findings regarding the EUS features of gastric schwannoma.

In conclusion, our examination of EUS images revealed that gastric schwannomas were heterogeneously hypoechoic lesions with distinct margins and marginal haloes that originate in the proper muscle layer. The echogenicity of these lesions was lower than that of the surrounding normal proper muscle layer, and cystic changes and calcifications were rare. Although more invasive procedures may be needed to obtain tissue samples in some cases, these EUS features may be helpful for differentiating gastric schwannomas from other mesenchymal tumors, especially GISTs.

\section{Conflicts of Interest}

The authors have no financial conflicts of interest. 


\section{Acknowledgments}

This work was supported by a 2-Year Research Grant of Pusan National University.

\section{REFERENCES}

1. Kim GH, Kim KB, Lee SH, et al. Digital image analysis of endoscopic ultrasonography is helpful in diagnosing gastric mesenchymal tumors. BMC Gastroenterol 2014;14:7.

2. Papanikolaou IS, Triantafyllou K, Kourikou A, Rösch T. Endoscopic ultrasonography for gastric submucosal lesions. World J Gastrointest Endosc 2011;3:86-94

3. Voltaggio L, Murray R, Lasota J, Miettinen M. Gastric schwannoma: a clinicopathologic study of 51 cases and critical review of the literature. Hum Pathol 2012;43:650-659.

4. Sarlomo-Rikala M, Miettinen M. Gastric schwannoma: a clinicopathological analysis of six cases. Histopathology 1995;27:355-360.

5. Fujiwara S, Nakajima K, Nishida T, et al. Gastric schwannomas revisited: has precise preoperative diagnosis become feasible? Gastric Cancer 2013;16:318-323

6. Kim GH, Park do Y, Kim S, et al. Is it possible to differentiate gastric GISTs from gastric leiomyomas by EUS? World J Gastroenterol 2009;15:3376-3381.

7. Jung MK, Jeon SW, Cho CM, et al. Gastric schwannomas: endosonographic characteristics. Abdom Imaging 2008;33:388-390.

8. Park HC, Son DJ, Oh HH, et al. Endoscopic ultrasonographic characteristics of gastric schwannoma distinguished from gastrointestinal stromal tumor. Korean J Gastroenterol 2015;65:21-26.

9. Zhong DD, Wang $\mathrm{CH}, \mathrm{Xu}$ JH, Chen MY, Cai JT. Endoscopic ultrasound features of gastric schwannomas with radiological correlation: a case series report. World J Gastroenterol 2012;18:7397-7401.

10. Yamada T, Ichikawa H. X-ray diagnosis of elevated lesions of the stomach. Radiology 1974;110:79-83.

11. Hou YY, Tan YS, Xu JF, et al. Schwannoma of the gastrointestinal tract: a clinicopathological, immunohistochemical and ultrastructural study of 33 cases. Histopathology 2006;48:536-545.

12. Larghi A, Verna EC, Ricci R, et al. EUS-guided fine-needle tissue acquisition by using a 19-gauge needle in a selected patient population: a prospective study. Gastrointest Endosc 2011;74:504-510.
13. Kim GH, Cho YK, Kim EY, et al. Comparison of 22-gauge aspiration needle with 22-gauge biopsy needle in endoscopic ultrasonography-guided subepithelial tumor sampling. Scand J Gastroenterol 2014;49:347-354

14. DeWitt J, Emerson RE, Sherman S, et al. Endoscopic ultrasound-guided Trucut biopsy of gastrointestinal mesenchymal tumor. Surg Endosc 2011;25:2192-2202

15. Melvin WS, Wilkinson MG. Gastric schwannoma. Clinical and pathologic considerations. Am Surg 1993;59:293-296.

16. Levy AD, Quiles AM, Miettinen M, Sobin LH. Gastrointestinal schwannomas: CT features with clinicopathologic correlation. AJR Am J Roentgenol 2005;184:797-802

17. Hong HS, Ha HK, Won HJ, et al. Gastric schwannomas: radiological features with endoscopic and pathological correlation. Clin Radiol 2008;63:536-542.

18. Takeda M, Amano Y, Machida T, Kato S, Naito Z, Kumita S. CT, MRI, and PET findings of gastric schwannoma. Jpn J Radiol 2012;30:602-605.

19. Ohno T, Ogata K, Kogure N, et al. Gastric schwannomas show an obviously increased fluorodeoxyglucose uptake in positron emission tomography: report of two cases. Surg Today 2011;41:1133-1137.

20. Okai T, Minamoto T, Ohtsubo K, et al. Endosonographic evaluation of c-kit-positive gastrointestinal stromal tumor. Abdom Imaging 2003;28:301-307.

21. Fujii Y, Taniguchi N, Hosoya Y, et al. Gastric schwannoma: sonographic findings. J Ultrasound Med 2004;23:1527-1530.

22. Appelman HD, Helwig EB. Sarcomas of the stomach. Am J Clin Pathol 1977;67:2-10.

23. Rösch T. Endoscopic ultrasonography in upper gastrointestinal submucosal tumors: a literature review. Gastrointest Endosc Clin N Am 1995;5:609-614

24. Palazzo L, Landi B, Cellier C, Cuillerier E, Roseau G, Barbier JP. Endosonographic features predictive of benign and malignant gastrointestinal stromal cell tumours. Gut 2000;46:88-92.

25. Agaimy A, Märkl B, Kitz J, et al. Peripheral nerve sheath tumors of the gastrointestinal tract: a multicenter study of 58 patients including NF1-associated gastric schwannoma and unusual morphologic variants. Virchows Arch 2010;456:411-422.

26. Fernández-Esparrach G, Sendino O, Solé M, et al. Endoscopic ultrasound-guided fine-needle aspiration and trucut biopsy in the diagnosis of gastric stromal tumors: a randomized crossover study. Endoscopy 2010:42:292-299. 